\title{
Medium-Chain Acyl-CoA Dehydrogenase Deficiency in Children with Non-Ketotic Hypoglycemia and Low Carnitine Levels
}

\author{
CHARLES A. STANLEY, ${ }^{(34)}$ DANIEL E. HALE, PAUL M. COATES, CAROLE L. HALL, \\ BARBARA E. CORKEY, WILLIAM YANG, RICHARD I. KELLEY, ELISA L. GONZALES, \\ JOHN R. WILLIAMSON, AND LESTER BAKER \\ Division of Endocrinology/Diabetes, [C.A.S., D.E.H., E.L.G., L.B.], Genetics [P.M.C.], and Metabolism \\ [W.Y.,R.I.K.] of the Department of Pediatrics and the Department of Biochemistry and Biophysics \\ [B.E.C.,J.W.R.J, University of Pennsylvania School of Medicine; The Children's Hospital of Philadelphia, \\ Philadelphia, Pennsylvania and the School of Chemistry, Georgia Institute of Technology [C.L.H.], Atlanta,
} Georgia, USA

\begin{abstract}
Summary
Three children in two families presented in early childhood with episodes of illness associated with fasting which resembled Reye's syndrome: coma, hypoglycemia, hyperammonemia, and fatty liver. One child died with cerebral edema during an episode. Clinical studies revealed an absence of ketosis on fasting (plasma beta-hydroxybutyrate $<\mathbf{0 . 4} \mathbf{~ m m o l e / l i t e r ) ~ d e s p i t e ~ e l e v a t e d ~ l e v e l s ~}$ of free fatty acids (2.6-4.2 mmole/liter) which suggested that hepatic fatty acid oxidation was impaired. Urinary dicarboxylic acids were elevated during illness or fasting. Total carnitine levels were low in plasma (18-25 $\mu$ mole/liter), liver (200-500 nmole/ $\mathrm{g}$ ), and muscle (500-800 nmole/g); however, treatment with $\mathrm{L}-$ carnitine failed to correct the defect in ketogenesis. Studies on ketone production from fatty acid substrates by liver tissue in vitro showed normal rates from short-chain fatty acids, but very low rates from all medium and long-chain fatty acid substrates. These results suggested that the defect was in the mid-portion of the intramitochondrial beta-oxidation pathway at the mediumchain acyl-CoA dehydrogenase step. A new assay for the electron transfer flavoprotein-linked acyl-CoA dehydrogenases was used to test this hypothesis. This assay follows the decrease in electron transfer flavoprotein fluorescence as it is reduced by acyl-CoA-acyl-CoA dehydrogenase complex. Results with octanoyl-CoA as substrate indicated that patients had less than $2.5 \%$ normal activity of medium-chain acyl-CoA dehydrogenase. The activities of short-chain and isovaleryl acyl-CoA dehydrogenases were normal; the activity of long-chain acyl-CoA dehydrogenase was one-third normal.

These results define a previously unrecognized inherited metabolic disorder of fatty acid oxidation due to deficiency of medium-chain acyl-CoA dehydrogenase. The carnitine deficiency in these patients appears to be a secondary consequence of their defect in fatty acid oxidation. It is possible that other patients with "systemic carnitine deficiency," who fail to respond to carnitine therapy, may also have defects in fatty acid oxidation similar to medium-chain acyl-CoA dehydrogenase deficiency.
\end{abstract}

Abbreviations

ACD, acyl-CoA dehydrogenase,

BOB, beta-hydroxybutyrate

CPT, carnitine palmityl transferase

$\mathrm{CPT}_{1}$, CPT activity on outer surface of mitochondria

ETF, electron transfer flavoprotein

FFA, free fatty acid
The oxidation of fatty acids for energy production not only plays an important role in working skeletal and cardiac muscle, but also is crucial for maintenance of fuel homeostasis during periods of fasting. During fasting, the utilization of fatty acids helps to conserve glucose for brain metabolism and the oxidation of fatty acids to ketones in the liver yields both energy for gluconeogenesis as well as fat-derived substrates that can be used by the brain to further spare glucose consumption. In the past several years, an increasing number of inherited defects in the pathway of fatty acid oxidation have been described. Depending on the degree of involvement of liver, heart, and skeletal muscle, the clinical features of these disorders range from recurrent episodes of fasting coma and hypoglycemia, which may mimic Reye's syndrome, to progressive muscle weakness resembling muscular dystrophy. In some of these disorders, such as carnitine palmityl-transferase $(2,8)$ and hydroxymethyl-glutaryl-CoA lyase deficiencies (27), the site of the metabolic defect has been identified. In others, such as type II glutaric aciduria (24) and the dicarboxylic acidurias $(1,4,13,23,31)$, the site of defect has not yet been established. One of the disorders where the site of defect remains uncertain is a heterogeneous group associated with deficiency of carnitine, a co-factor required for transport of longchain fatty acids into mitochondria. At least two clinical syndromes appear to be associated with carnitine deficiency: a systemic form (16) and a myopathic form (9). Carnitine replacement therapy has sometimes been effective (5), but frequently is not (3), suggesting that the primary abnormality in many of these cases is not in carnitine biosynthesis $(10,11)$.

The present report describes two families with a defect in fatty acid oxidation associated with low plasma and tissue carnitine levels in which carnitine deficiency is not the cause, but instead may be the consequence of impaired fatty acid oxidation. The affected children in these two families presented in early childhood with life-threatening episodes of fasting coma and hypoglycemia that resembled Reye's syndrome. Clinical studies revealed an absence of ketosis during fasting, which suggested that hepatic fatty acid oxidation was impaired. Because there were no signs of muscle weakness, liver biopsies were obtained to analyze the pathway of fatty acid oxidation. These studies demonstrated a defect in intramitochondrial beta-oxidation at the medium-chain acyl-CoA dehydrogenase step. A specific deficiency of this enzyme was confirmed using a new assay that was developed for use in small samples of unfractionated tissue. Subsequent studies have shown that this defect can now also be demonstrated in cultured skin fibroblasts (6). 
PATIENTS

Patient $M-1$. This female patient was first admitted to the Children's Hospital of Philadelphia at 13 months of age because of coma. She had been the $2660 \mathrm{~g}$ product of a normal pregnancy and, except for frequent ear infections, had been well. Her growth and development had also been normal. Four days before admission she developed symptoms of a viral illness with low grade fever, irritability, mild diarrhea, and intermittent vomiting. On the morning of admission she was found unarousable in bed with labored breathing. She stopped breathing on the way to another hospital and was given mouth-to-mouth ventilation. In the emergency room she had a generalized seizure and received a large dose of diazepam. She was stabilized and transported to the Children's Hospital of Philadelphia. On arrival she was obtunded, responded only to painful stimuli, and required mechanical ventilation. Physical examination was normal; the liver was not enlarged. Laboratory studies showed a spinal fluid glucose of $23 \mathrm{mg} / \mathrm{dl}$ (1.3 mmole/liter); normal serum sodium, potassium, and chloride; bicarbonate, $11 \mathrm{mmole} / \mathrm{liter}$; urea nitrogen, $14 \mathrm{mg} / \mathrm{dl}(5.0 \mathrm{mmole} /$ liter $)$; blood ammonia, $52 \mu \mathrm{g} / \mathrm{dl}$ (30 $\mu$ mole/liter); hemoglobin, $12.3 \mathrm{~g} / \mathrm{dl}$ ( $7.7 \mathrm{mmole} / \mathrm{liter})$; and white blood count, $21,700 / \mathrm{mm}^{3}\left(21.7 \times 10^{9} /\right.$ liter $)$; and a small amount of ketones in the urine. She was treated with intravenous dextrose and fluids and began to improve slowly. On the third day, when the intravenous fluids were stopped and nasogastric feedings were begun she became obtunded again and had a cardiorespiratory arrest. At this time the liver was noted to be firm and enlarged to $4 \mathrm{~cm}$ below the costal margin. The plasma glucose was $34 \mathrm{mg} / \mathrm{dl}$ ( $1.9 \mathrm{mmole} / \mathrm{liter}$ ); serum bicarbonate, 16 mmole/liter; and blood ammonia, $138 \mu \mathrm{g} / \mathrm{dl}(81 \mu \mathrm{mole} / \mathrm{liter})$. A urine specimen at the time of this episode showed very elevated concentrations of medium-chain dicarboxylic acids (see "Results"). She was successfully resuscitated and again treated with dextrose and fluids. She gradually improved and was discharged 12 days later, apparently fully recovered. A blood specimen obtained at the time of discharge showed a very low plasma total carnitine level ( $7 \mu$ mole/liter; normal 40-60). At age 21 months, she was readmitted for lethargy and vomiting after 3 days of moderate diarrhea. She responded well to intravenous fluids and dextrose and was discharged after 1 day in the hospital. Apart from these two episodes, she has remained well. At age $2 \% 12$ her physical examination, including muscle strength and intellectual development, is normal.

The patient's mother, father, and 6-year-old sister have never had similar illnesses. The mother and father are unrelated and have total plasma carnitine levels that are normal (50 and 46 $\mu$ mole/liter, respectively).

Patient $B$ - 1 . This $2 / 12$-year-old female was admitted to another hospital for coma and hypoglycemia. She had been well the previous day and attended a family picnic. On the morning of admission she was found convulsing in bed and on arrival in the other hospital was pale, bradycardic, and unresponsive to painful stimuli. The liver was palpated at the costal margin. Laboratory studies included a plasma glucose of $2 \mathrm{mg} / \mathrm{dl}(0.1 \mathrm{mmole} / \mathrm{liter})$; normal sodium, potassium, and chloride; bicarbonate $19 \mathrm{mmole} /$ liter; urea nitrogen, $32 \mathrm{mg} / \mathrm{dl}$ ( $11 \mathrm{mmole} / \mathrm{liter}$ ); and small ketonuria. She improved slightly after intravenous dextrose, but developed persistent seizures and was transferred to the Children's Hospital of Philadelphia after $16 \mathrm{~h}$. She had no reflexes or movement and required mechanical ventilation. She died with an isoelectric electroencephalogram 2 days later. Additional laboratory studies at Children's Hospital of Philadelphia showed a slightly elevated blood ammonia of $82 \mu \mathrm{g} / \mathrm{dl}(48 \mu \mathrm{mole} / \mathrm{liter})$; transaminase, $68 \mathrm{U} / \mathrm{ml}\left(0.54 \mu \mathrm{mol} \cdot \mathrm{sec}^{1} \cdot\right.$ liter $\left.^{-1}\right)$; and a negative screening test for ethanol and aspirin. No abnormal increases in urine organic acids were found by gas chromatography (see "Results"). A frozen needle liver biopsy showed panlobular accumulation of fat droplets in hepatocytes, which was interpreted to be consistent with Reye's syndrome. Postmortem examination revealed brain swelling with uncal herniation as the cause of death.
The past medical history was remarkable only for a prior unexplained episode of hypoglycemia at 6 months of age. Growth, development, and muscle strength had been normal. Specimens of liver and skeletal muscle were obtained soon after death. Total carnitine levels in these tissues were found to be low (see "Results") and suggested the possibility of an inherited metabolic defect in fatty acid oxidation.

Patient B-2. Patient B-2 is the oldest brother of patient B-1. At age $23 / 12$, he had an episode of coma and hypoglycemia after sleeping late one morning. He cried out and was found unresponsive by his parents. He was taken to an emergency room where he was noted to be staring and unresponsive with arms and legs stiffly flexed. Laboratory studies showed plasma glucose, $8 \mathrm{mg} / \mathrm{dl}$ (0.44 mmole/liter); normal serum sodium, potassium and chloride; serum bicarbonate, $19 \mathrm{mmole} / \mathrm{liter}$; and moderate ketonuria. He was transferred to Children's Hospital of Philadelphia where additional laboratory tests showed an elevated blood ammonia (185 $\mu \mathrm{g} / \mathrm{dl} ; 109 \mu$ mole/liter) compared to a control ( $150 \mu \mathrm{g} / \mathrm{dl} ; 88 \mu \mathrm{mole} /$ liter $)$, slightly elevated transaminase $\left(64 \mathrm{U} / \mathrm{ml} ; 0.5 \mu \mathrm{mol} \cdot \mathrm{s}^{-1} /\right.$ liter $\left.^{-1}\right)$, and normal prothrombin time. The liver was not enlarged. He had several seizures after treatment was started with intravenous fluids. He improved slowly over 3 days. One week later, he tolerated a fast of $36 \mathrm{~h}$ without developing hypoglycemia (lowest plasma glucose, $58 \mathrm{mg} / \mathrm{dl} ; 3.2$ mmole/liter) or symptoms. Because the episode of coma had occurred after a longer period of overnight fasting than was usual for him, the parents were advised to avoid letting him fast more than $12 \mathrm{~h}$. He has had no further episodes of coma. At age 10 years, he appears physically and developmentally normal and has no signs of muscle weakness. His total plasma carnitine level is low ( $25 \mu$ mole/liter $)$.

The two other siblings in the B family have never had episodes of coma: a boy $2-2 / 12$ years old and a girl $11-5 / 12$ years old. They have normal physical examinations and no evidence of weakness. Total plasma carnitine levels are low in the brother $(25 \mu \mathrm{mole} /$ liter) and normal in the sister ( $40 \mu$ mole/liter). The parents are unrelated and have been healthy.

\section{MATERIALS AND METHODS}

Studies of fasting adaptation and response to oral L-carnitine therapy were carried out in the Clinical Research Center at the Children's Hospital of Philadelphia. Signed consent for these studies was obtained from the parents of the patients. These studies were approved by the Committee for Protection of $\mathrm{Hu}$ man Subjects of the Children's Hospital of Philadelphia. Studies of fasting adaptation were begun after an 8 p.m. bedtime snack and plasma glucose levels were monitored frequently to avoid severe hypoglycemia. During these fasts, patients were encouraged to drink water or noncaloric beverages. Blood samples were obtained from indwelling pediatric scalp vein needles kept patent by intermittent flushing with heparinized saline. Urine samples were collected on ice in containers with $5 \mathrm{ml}$ of sodium hydroxide for measurement of dicarboxylic acids.

Samples of liver tissue and rectus abdominis muscle weighing $200-400 \mathrm{mg}$ were obtained at the time of diagnostic surgical open liver biopsy. Liver and muscle biopsies were also obtained from the younger brother of patients B-1 and B-2 because of parental concern that he might be at risk for developing the same illness as his deceased sister.

Plasma glucose was determined using a Beckman glucose analyzer. Blood lactate and plasma FFA and BOB levels were determined as previously described (28). Plasma and tissue carnitine levels were measured with a radioenzymatic method (19). Urine organic acids were measured as the trimethylsilyl derivatives by gas liquid chromatography. Identity of derivatives was confirmed by gas chromatography-mass spectrometry. L-Carnitine was generously provided by Dr. Monroe Klein, Sigma Tau, Inc., Holmdel, NJ.

In vitro capacity for fatty acid oxidation to ketones was measured using homogenates of freshly-obtained liver tissue in $0.1 \mathrm{ml}$ final volume containing $4 \mathrm{mg}$ tissue, $10 \mathrm{mM}$ potassium phos- 
phate buffer ( $\mathrm{pH} 7.4), 137 \mathrm{mM} \mathrm{KCl}, 10 \mathrm{mM}$ alpha-ketoglutarate, $1 \mathrm{mM} \mathrm{MgCl}, 1 \mathrm{mM} \mathrm{ATP}, 0.5 \mathrm{mM}$ EDTA, $0.05 \mathrm{mM}$ coenzyme $\mathrm{A}, 1 \%$ bovine serum albumin, and different fatty acid substrates at concentrations that gave maximal rates of ketogenesis in studies of guinea pig and rat liver $(17,29)$. Incubations were run in duplicate in room air at $37^{\circ} \mathrm{C}$ for $30 \mathrm{~min}$. Where used, the concentration of L-carnitine was $0.5 \mathrm{mM}$. With alpha-ketoisocaproate as substrate, the incubation was modified to contain 24 $\mathrm{mM} \mathrm{KHCO} 3$ and run in a $95 \% \mathrm{O}_{2}: 5 \% \mathrm{CO}_{2}$ atmosphere. The $\mathrm{BOB}$ and acetoacetate produced were assayed by an enzymatic method (32) modified for a fluorometer. As reported by Krebs (17), the inclusion of alpha-ketoglutarate or other oxidizable substrate in this system maintains mitochondrial ATP levels and helps to maximize the flow of fatty acid carbon to ketones rather than $\mathrm{CO}_{2}$ : with $\left[1-{ }^{14} \mathrm{C}\right]$ palmitate, greater than $95 \%$ of the fatty acid oxidized appears in ketones and less than $5 \%$ as $\mathrm{CO}_{2}$.

Carnitine palmityl-transferase (E.C.2.3.1.21) activity in fresh whole homogenate of liver $\left(\mathrm{CPT}_{1}\right)$ was determined using a modification (28) of the "forward" isotopic reaction conditions described by McGarry et al. (20). The inhibition of the reaction by $20 \mu \mathrm{M}$ malonyl-CoA was determined to confirm that the assay reflected primarily $C P \mathrm{C}_{1}$ activity.

The activities of four different ACD enzymes in liver tissue (short-chain, medium-chain, long-chain, and isovaleryl ACD: E.C.1 3.9.9.2;3.+10) were determined by directly measuring the extent of decrease in oxidized ETF fluorescence without a final electron acceptor in the presence of excess amounts of the specific acyl-CoA substrates for these enzymes. Samples of liver tissue stored at $-40^{\circ} \mathrm{C}$ were homogenized and sonicated to release mitochondrial ACD activity into the $40,000 \times g$ supernatant. Aliquots containing $0-1000 \mu \mathrm{g}$ liver protein were added to cuvettes containing $0.2 \mathrm{nmole}$ purified porcine ETF in $1.0 \mathrm{ml} 20$ $\mathrm{mM}$ potassium phosphate buffer ( $\mathrm{pH}$ 7.6) in a fluorometer using excitation and emission wavelengths of $405 \mathrm{~nm}$ and $490 \mathrm{~nm}$, respectively. The decrease in fluorescence after the addition of either butyryl-CoA, octanoyl-CoA, palmityl-CoA, or isovalerylCoA $(25 \mu \mathrm{M})$ was recorded. The maximal decrease of ETF fluorescence was determined by subsequent addition of excess purified porcine liver medium-chain, short-chain, or isovalerylCoA dehydrogenase. This assay is based on the observation that when ACD activity in the sample is limiting, there is a rapid but incomplete reduction in oxidized ETF that remains stable for more than $30 \mathrm{~min}$ (14). The extent of ETF reduction, as a \% of maximal, increases with increasing amounts of $A C D$, in a concentration-dependent saturation curve.

This assay avoids the problems of high backgrounds, which make the dye-reduction (14) or labeled-substrate (26) assays difficult to use with samples of frozen tissue where an initial purification of the ACD enzymes is unfeasible. The assay does not quantitate enzyme activity in traditional terms of velocity of substrate disappearance or product formation. Instead, a comparison of the saturation curves for different samples permits an estimation of the relative content of $A C D$ in a manner analogous to measurement of the relative potency of different hormone preparations in radioimmunoassays. Enzyme activity is wellpreserved in frozen tissue and in autopsy samples taken several hours postmortem. The assay has been successfully used in cultured skin fibroblasts (6) and, recently, in white blood cells. A more complete description of the assay is in preparation by Dr. Hall who also kindly provided the ETF and porcine ACD preparations.

\section{RESULTS}

Because the illnesses in the three patients, M-1, B-1, and B-2, seemed to have occurred in situations of fasting stress, the changes in levels of circulating metabolic fuels during fasting were examined. Figure 1 shows the concentrations of plasma glucose, FFA, BOB, and blood lactate during fasts in patients $\mathrm{M}-1$ and B-2. The levels of urinary organic acids and plasma carnitine during these studies are shown in Tables 1 and 2 . The data from both patients $\mathrm{M}-1$ and B-2 revealed a severe limitation

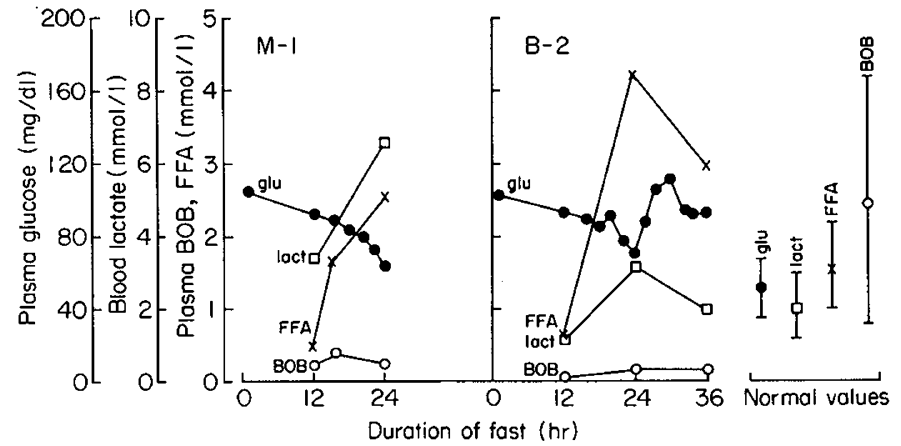

Fig. 1. Responses to fasting in patients $M-1$ and $B-2$. Shown are levels of glucose (glu), beta-hydroxybutyrate (BOB), free fatty acids (FFA), and lactate (lact). Normal values indicate means and ranges found in infants after 24-h fasts (Ref 28).

Table 1. Urinary beta-hydroxybutyric acid and medium-chain dicarboxylic acids in patients $M-1, B-1$, and $B-2$ ( $\mathrm{mg} / \mathrm{g}$ creatinine)

\begin{tabular}{llcccc}
\hline Patient & Status & $\begin{array}{c}\text { Beta- } \\
\text { hydroxy- } \\
\text { butyric }\end{array}$ & $\begin{array}{c}\text { Adipic } \\
\left(\mathrm{C}_{6}\right)\end{array}$ & $\begin{array}{c}\text { Suberic } \\
\left(\mathrm{C}_{8}\right)\end{array}$ & $\begin{array}{c}\text { Sebacic } \\
\left(\mathrm{C}_{10}\right)\end{array}$ \\
\hline M-1 & Sick & 560 & 6740 & 8390 & 8860 \\
& Fed & $<10$ & $<20$ & $<20$ & $<20$ \\
& Fasted & 20 & 70 & 160 & 200 \\
B-1 & Pre-demise & $<10$ & $<20$ & $<20$ & $<20$ \\
B-2 & Fed & 10 & $<20$ & 10 & $<10$ \\
& Fasted & 40 & $<20$ & 170 & 50 \\
Controls & & & & & \\
1 & Fasted & 750 & $<20$ & $<20$ & $<20$ \\
2 & Fasted & 400 & $<20$ & $<20$ & $<20$ \\
3 & Fasted & 420 & $<20$ & $<20$ & $<20$ \\
Isovaleric- & Fasted & 1420 & 270 & $<20$ & $<20$ \\
acidemia & & & & & \\
\hline
\end{tabular}

in hepatic ketogenesis. Patient M-1 developed signs of lethargy and vomiting, which resembled those of her spontaneous episodes of illness at $24 \mathrm{~h}$ of fasting. At this time, her plasma glucose level had only fallen to $64 \mathrm{mg} / \mathrm{dl}$ (3.6 mmole/liter). Her plasma FFA levels rose from 12 to $24 \mathrm{~h}$ to reach an unusually high value of $2.6 \mathrm{mmole} / \mathrm{liter}$. Her plasma levels of $\mathrm{BOB}$ remained less than $0.4 \mathrm{mmole} / \mathrm{liter}$, indicating a limitation in hepatic fatty acid oxidation to ketones. A very similar pattern was observed in patient B-2, although he tolerated $36 \mathrm{~h}$ of fasting without developing the severe symptoms seen in $\mathrm{M}-1$. Coincident with a transient fall in plasma glucose level to $70 \mathrm{mg} / \mathrm{dl}(3.9 \mathrm{mmole} /$ liter) at $24 \mathrm{~h}$, his plasma FFA concentration reached $4.2 \mathrm{mmole} /$ liter and remained very elevated at $2.95 \mathrm{mmole} / \mathrm{liter}$ at $36 \mathrm{~h}$. He demonstrated a limitation in hepatic ketogenesis, similar to patient $\mathrm{M}-1$, with plasma $\mathrm{BOB}$ levels remaining below 0.3 mmole/liter throughout the $36 \mathrm{~h}$. Blood lactate concentrations were slightly elevated in both of these patients, rising to 6.6 mmole/liter at $24 \mathrm{~h}$ in $\mathrm{M}-1$ and reaching $3.2 \mathrm{mmole} / \mathrm{liter}$ at 24 $\mathrm{h}$ in B-2 before falling to $1.9 \mathrm{mmole} / \mathrm{liter}$ at $36 \mathrm{~h}$. Of interest is the fact that plasma triglyceride levels remained normal in B-2 during the fast $(0.60,0.78$, and $0.52 \mathrm{~g} /$ liter at 12,24 , and $48 \mathrm{~h})$ despite his impairment in hepatic fatty acid oxidation. The younger brother of patients B-1 and B-2 was able to increase hepatic ketone production with plasma levels of $\mathrm{BOB}$ after a 24-h fast of $3.3 \mathrm{mmole} / \mathrm{liter}$; glucose, $55 \mathrm{mg} / \mathrm{dl}$ ( $3.1 \mathrm{mmole} / \mathrm{liter})$; and FFA, 3.3 mmole/liter.

Table 1 shows the urinary concentrations of $\mathrm{BOB}$ and medium-chain dicarboxylic acids in patients $\mathrm{M}-1, \mathrm{~B}-1$, and $\mathrm{B}-2$ compared to three control children and one patient with isovaleric-acidemia. No abnormalities were found in patients $M-1$ and B-2 when they were well or in patient B-1 2 days after the onset 
Table 2. Plasma carnitine concentrations during fasting in patients $M-I$ and B-2 compared to control children ( $\mu$ mole/liter mean \pm S.D.)

\begin{tabular}{|c|c|c|c|c|c|c|}
\hline \multirow[b]{2}{*}{ Patient } & \multirow[b]{2}{*}{ Hour } & \multirow[b]{2}{*}{ Free carnitine } & \multicolumn{3}{|c|}{ Esterified carnitine } & \multirow[b]{2}{*}{ Total carnitine } \\
\hline & & & $\begin{array}{c}\text { Acid } \\
\text { soluble }\end{array}$ & $\begin{array}{c}\text { Acid } \\
\text { insoluble }\end{array}$ & $\begin{array}{c}\text { Total } \\
\text { esterified }\end{array}$ & \\
\hline \multirow[t]{2}{*}{ M-1 } & 12 & 11 & 14 & 0 & 14 & 25 \\
\hline & 24 & 21 & 32 & 17 & 49 & 70 \\
\hline \multirow[t]{4}{*}{ B-2 } & 0 & 18 & & & 0 & 18 \\
\hline & 12 & 14 & & & 6 & 20 \\
\hline & 24 & 22 & & & 11 & 33 \\
\hline & 36 & 20 & & & 12 & 32 \\
\hline \multirow[t]{2}{*}{$\begin{array}{l}\text { Controls } \\
\qquad(n=10)\end{array}$} & Fed & $42 \pm 1$ & & & $6 \pm 3$ & $\begin{array}{l}49 \pm 6 \\
\quad \text { (range, 40-60) }\end{array}$ \\
\hline & Fasted & $28 \pm 9.5$ & & & $21 \pm 9.5$ & $\begin{array}{l}49 \pm 6 \\
\quad \text { (range, 39-60) }\end{array}$ \\
\hline
\end{tabular}

of her illness, however, urinary dicarboxylic acids were markedly elevated in the patients when they were acutely ill (M-1) or fasted (M-1 and B-2). An increase in the urinary excretion of these compounds is normally seen during the accelerated fatty acid oxidation of fasting; however, in normal subjects, the ratio of urinary $\mathrm{BOB}$ to any of the dicarboxylic acids greatly exceeds unity. This ratio was reversed in patients $M-1$ and $B-2$, in accord with their impairment in ketogenesis demonstrated on fasting (Fig. 1). No other abnormal urinary organic acids, including ethylmalonic (22) and (omega-1)-hydroxy-fatty acids (18) were found in the patients.

Table 2 shows the plasma concentrations of free and esterified carnitine during the fasting studies shown in Figure 1 in patients M-1 and B-2. In both patients, total plasma carnitine levels were low in the postabsorptive state, after a 12-h fast, compared with the controls. In both M-1 and B-2, the levels of total carnitine rose during the fast, with most of the change due to an increase in esterified carnitine. In patient $\mathrm{M}-1$, fractionation of the esterified carnitine showed that both long-chain acyl (acid insoluble) and short- and medium-chain acyl carnitine (acid soluble) esters were markedly elevated at 24 hours. In normal children (Table 2) and adults, total plasma carnitine levels change little with fasting, but the esterified fraction increases in association with accelerated fatty acid oxidation and ketone synthesis. In view of later findings of a defect in mitochondrial beta-oxidation in patients $\mathrm{M}-1$ and $\mathrm{B}-2$, it is possible that their increase in plasma esterified carnitine reflects an accumulation of unoxidized fatty acid intermediates during fasting.

Table 3 shows the concentration of total carnitine in liver and rectus abdominis biopsies obtained from patient $\mathrm{M}-1$ and $\mathrm{B}-2$, and in these tissues obtained from patient B-1 postmortem. In all three affected patients (M-1, B-1, and B-2), carnitine levels were only $20-40 \%$ of normal values in both liver and skeletal muscle. Tissue levels of carnitine in the unaffected brother of patients B-1 and B-2 were normal, although his plasma level of carnitine was reduced ( $25 \mu \mathrm{mole} / \mathrm{liter})$.

Patients M-1 and B-2 were given carnitine for 2 months to see whether treatment would improve their capacity for hepatic fatty acid oxidation. Both received $75-100 \mathrm{mg} \cdot \mathrm{kg}^{-1} \cdot$ day $^{-1} \mathrm{~L}$-carnitine orally, divided into three doses. Although plasma carnitine levels were normal (B-2) or above normal (M-1) on treatment, fasting responses were essentially identical to those shown in Fig. 1; neither patient was able to increase plasma BOB levels despite elevated concentrations of FFA.

Light microscopy of the liver biopsies from patient M-1 and B-2 revealed a small amount of neutral fat in hepatocytes. In M1 , there were a few round cells in the portal tracts and, rarely, within the liver lobules. Electron microscopy showed a considerable number of mitochondria containing lamellar structures [Type A crystals (30)], especially in patient M-1. The implication of this finding is uncertain; these structures can be seen in normal liver, although to a lesser degree than found in these patients.
Table 3. Total carnitine concentration in liver and skeletal muscle from patients $M-1, B-1, B-2$, compared to control children (nmole/g wet weight, mean $\pm S . D$.)

\begin{tabular}{lcc}
\hline \multicolumn{1}{c}{ Patient } & Liver & Muscle \\
\hline $\mathrm{M}-1$ & 500 & 800 \\
$\mathrm{~B}-1$ & 275 & 510 \\
$\mathrm{~B}-2$ & 201 & 540 \\
Brother of B-1 and B-2 & 1330 & 2800 \\
Controls & $1200 \pm 300$ & 3200 \\
& (range, $730-1800)$ & $(n=1)$ \\
& $(n=11)$ & \\
\hline
\end{tabular}

Carnitine palmityl-transferase activity in fresh liver tissue from patient M-1 $\left(17.3 \mu \mathrm{mole} \cdot \mathrm{h}^{-1} \cdot \mathrm{g}^{-1}\right)$ and B-2 $\left(21.4 \mu \mathrm{mol} \cdot \mathrm{h}^{-1} \cdot \mathrm{g}^{-1}\right)$ was similar to that found in four controls (mean, $13.5 \mu$ mole. $\mathrm{h}^{-1} \cdot \mathrm{g}^{-1}$; range, 9.7-19). In both patients and controls, $20 \mu \mathrm{M}$ malonyl-CoA inhibited activity by $72-85 \%$, indicating that the assay measured primarily the enzyme activity on the outer surface of the inner mitochondrial membrane $\left(\mathrm{CPT}_{1}\right)$.

Because carnitine therapy failed to correct the defect in hepatic ketogenesis in patients M-1 and B-2, it appeared that the low levels of carnitine in these patients might not be the primary cause of their impaired fatty acid oxidation. In order to test directly the functional integrity of the hepatic oxidative pathway, rates of ketone synthesis from different fatty acid substrates were measured in fresh homogenates of liver tissue from patients $M$ 1 and B-2. Table 4 compares the results in these two patients with three control children aged 2-12.

From the data shown in Table 4 it is clear that ketone synthesis was markedly limited from all of the medium and long-chain fatty acid substrates in the two affected patients, M-1 and B-2, but ketogenesis from the short-chain fatty acid, butyrate, and from the branched-chain ketoacid, alpha-ketoisocaproate was normal. This indicates that the defect in these patients lies proximal to the last cycle of beta-oxidation and the HMG-CoA pathway. The fact that ketone synthesis from palmityl-carnitine was low indicates that the defect is distal to the carnitinerequiring $\mathrm{CPT}_{1}$ step and that the defect is not due to a deficiency of carnitine. A defect in acyl-carnitine transport or in $\mathrm{CPT}_{2}$ activity is also excluded because oxidation of octanoate, which is thought to enter mitochondria as the free acid and then be activated with coenzyme A, was also limited in M-1 and B-2. These results, therefore, suggest that the defect in these patients resides within the mitochondrial beta-oxidation pathway at the level of medium-chain fatty acyl-CoA oxidation. The site of defect appears to be the same in both families, although patient M-1 had slightly lower values for ketogenesis from long and medium-chain substrates than did patient B-2. This suggestion that patient M-1 may be more severely affected is consistent with the observation that she became symptomatic on fasting whereas patient B-2 did not. 
Table 4. Ketone synthesis from fatty acid substrates in liver homogenates from patient $M-1$ and $B-2(\mu m o l e / h / g)$

\begin{tabular}{|c|c|c|c|c|c|}
\hline \multirow[b]{2}{*}{ Substrate } & \multicolumn{2}{|c|}{ Patients } & \multicolumn{3}{|c|}{ Controls } \\
\hline & M-1 & B-2 & 1 & 2 & 3 \\
\hline $1 \mathrm{mM}$ palmitate plus carnitine & 2.0 & 3.9 & 7.5 & 10.6 & 8.2 \\
\hline $0.5 \mathrm{mM}$ palmityl-CoA plus carnitine & 2.4 & 4.4 & 9.3 & 10.7 & 11.0 \\
\hline $0.5 \mathrm{mM}$ palmityl-carnitine & 3.8 & 5.2 & 14.4 & 17.9 & 11.2 \\
\hline $2 \mathrm{mM}$ octanoate & 3.0 & 4.8 & 21.7 & 30.0 & 14.8 \\
\hline 2 mM octanoyl-CoA plus carnitine & 2.6 & 4.8 & 12.1 & 13.6 & 9.9 \\
\hline $2 \mathrm{mM}$ octanoyl-carnitine & 2.6 & 3.6 & 22.6 & 19.6 & 19.3 \\
\hline $10 \mathrm{mM}$ butyrate & 20.1 & 22.2 & 19.8 & 31.0 & 12.8 \\
\hline $1 \mathrm{mM}$ alpha-ketoisocaproate & 8.2 & 7.5 & 8.1 & 16.0 & 7.4 \\
\hline None & 0.8 & 3.0 & 3.0 & 5.0 & 5.7 \\
\hline
\end{tabular}
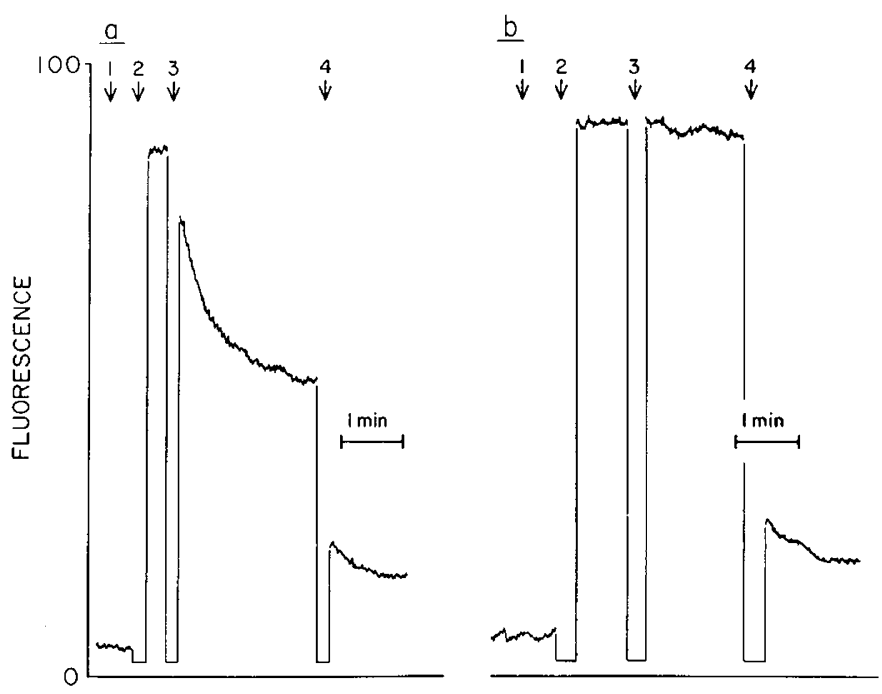

Fig. 2. Fluorometer tracings of medium-chain acyl-CoA dehydrogenase assay in liver from $(a)$ control and $(b)$ patient B-2. Vertical scale is in arbitrary units of fluorescent emission. Arrow $(l)$ is recording after addition of $100 \mu \mathrm{g}$ protein; (2) addition of ETF; (3) addition of octanoyl$\mathrm{CoA} ;(4)$ addition of an excess of porcine medium-chain acyl-CoA dehydrogenase. Plateaus after (2), (3), and (4) were used to calculate $\%$ of maximal ETF reduction due to enzyme activity in sample. For details, see "Materials and Methods."

Three of the four steps in the beta-oxidation cycle involve enzymes which are specific for fatty acids of different chain lengths (see "Discussion"). We hypothesized that deficiency of the medium-chain ACD might be present in the three affected patients, because this enzyme has the appropriate chain-length specificity to account for the findings with liver homogenates. To test this hypothesis, the activities of four ACD enzymes were evaluated in frozen liver tissue obtained at biopsy from patients M-1 and B-2 and at postmortem in patient B-1.

Figure 2 illustrates the assay of medium-chain ACD activity in patient B-2 and a control with tracings of the fluorometer records obtained using octanoyl-CoA as substrate. The \% maximal reduction of ETF was calculated by dividing the change in fluorescence after addition of substrate by the total change in fluorescence after subsequent addition of purified porcine medium-chain ACD. As shown, $100 \mu \mathrm{g}$ of liver protein from patient B-2 gave only $5 \%$ of maximal ETF reduction compared to $55 \%$ with the same amount of liver protein from the control.

Figure 3 shows the combined data on medium-chain $\mathrm{ACD}$ activity in the three patients, in the unaffected brother of patients B-1 and B-2, and in three other controls as the \% maximal ETF reduction found with different amounts of liver protein. In the controls, the data fall along a saturation curve with $25-30 \%$ maximal reduction at approximately $75 \mu \mathrm{g}$ protein. In contrast, in all three patients, no change in ETF fluorescence above

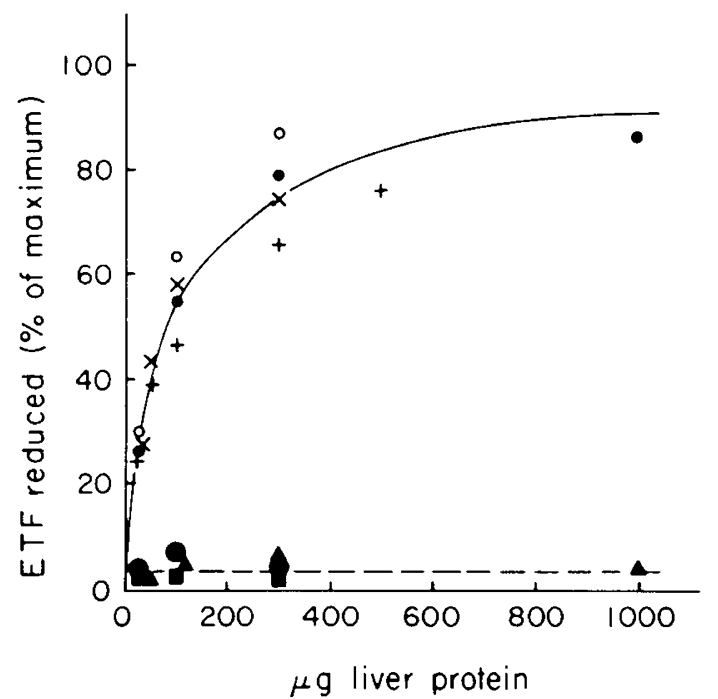

Fig. 3. Medium-chain acyl-CoA dehydrogenase activity in liver tissue

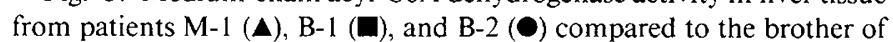
$\mathrm{B}-1$ and $\mathrm{B}-2(+)$ and three other controls $(\times, \bullet, O)$. Shown are the effects of different amounts of liver protein on the reduction of ETF fluorescence with octanoyl-CoA as substrate expressed as the \% of maximum achieved with excess porcine dehydrogenase.

background was seen with up to $1000 \mu \mathrm{g}$ of protein. Compared to the saturation curve in controls, this indicates that mediumchain ACD activity in liver from the three patients was less than $2.5 \%$ of normal. Mixing experiments showed no evidence of an enzyme inhibitor in patient livers.

Figure 4 shows the results of assays for the short-chain, isovaleryl, and long-chain ACD enzymes in patient and control liver. In controls, $50 \%$ maximal ETF reduction required more protein with butyryl-CoA $(150 \mu \mathrm{g})$, isovaleryl-CoA $(175 \mu \mathrm{g})$, and palmityl-CoA $(300 \mu \mathrm{g})$ as substrate than with octanoyl-CoA $(75 \mu \mathrm{g})$. This is consistent with the finding that the activity of mediumchain ACD is greater than that of the other ACD enzymes in porcine liver (14). The activity of long-chain ACD (panel c) in the patients' liver was only about one-third that of the controls. The higher activity in controls with palmityl-CoA as substrate probably reflects some contribution from the medium-chain $A C D$ enzyme to total long-chain ACD activity. As indicated by the data in Figure $4 a$ and $b$, short-chain and isovaleryl ACD enzyme activities were the same in patients and controls. These results demonstrate that the deficiency in the three patients is specific for medium-chain ACD activity.

\section{DISCUSSION}

The results of these studies define a metabolic disorder of mitochondrial fatty acid oxidation associated with deficient activity of medium-chain acyl-CoA dehydrogenase. This disorder 

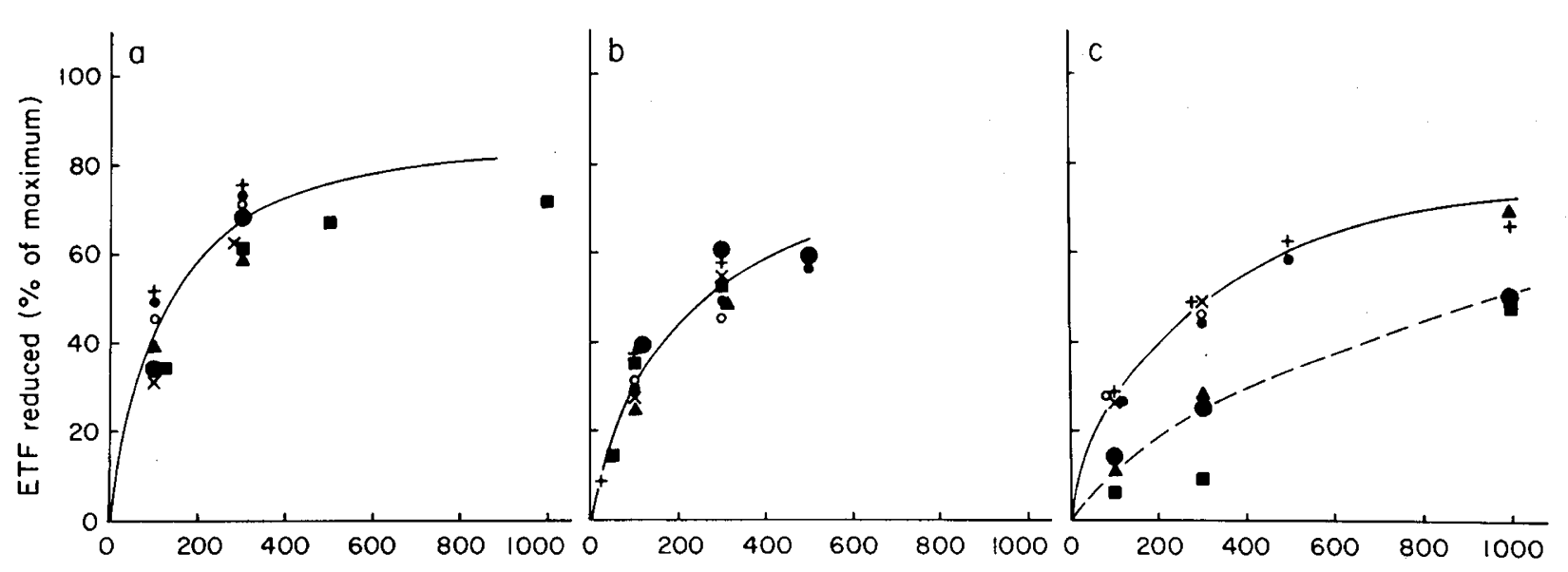

$\mu \mathrm{g}$ liver protein

Fig. 4. Short-chain $(a)$, isovaleryl $(b)$, and long-chain $(c)$ acyl-CoA dehydrogenase activity in liver tissue from the patients and controls shown in Figure 3. Symbols and expression of results are the same as noted in the legend to Figure 3. Substrates for the dehydrogenases were butyryl-CoA ( panel $a$ ), isovaleryl-CoA ( panel $b$ ) and palmityl-CoA ( panel $c$ ).

was demonstrated in three affected children (patients $\mathrm{M}-1, \mathrm{~B}-1$, and B-2) in two unrelated families. The clinical features in these patients include episodes of coma and hypoglycemia in infancy, apparently provoked by fasting stress. The presence of the metabolic defect was most readily demonstrated by the inability of affected patients to develop ketosis during fasting. Additional abnormalities included an increase in urinary excretion of dicarboxylic acids and decrease in beta-hydroxybutyrate under fasting stress; and reduced concentrations of carnitine in plasma, liver, and skeletal muscle.

Studies in liver tissue from the two surviving patients (M-1 and B-2) showed decreased oxidation of all long and mediumchain fatty acid substrates, but normal oxidation of short and branched-chain substrates. These results suggested that the site of defect was within the beta-oxidation cycle at the level of medium-chain acyl-CoA metabolism. All three affected patients (M-1, B-1, and B-2) were deficient in medium-chain ACD activity.

Medium-chain ACD is one of five or more related intramitochondrial flavin-containing enzymes that dehydrogenate fatty acyl-CoA esters at the alpha and beta carbons as the first step in the beta-oxidation cycle (14). The reaction reduces the dehydrogenase-bound flavin, which is then reoxidized by electron transfer to a second flavin protein, electron transfer flavoprotein, and subsequently to the electron transport chain. The alpha, beta-unsaturated fatty acyl-CoA produced in this reaction continues through the remaining three steps of the beta-oxidation cycle and, after the removal of an acetyl-CoA moiety, the fatty acyl-CoA re-enters the cycle shortened by two carbons. There are three $A C D$ enzymes that are specific for different ranges of fatty acid chain length: long-chain $A C D$, medium-chain $A C D$, and short-chain ACD. Two other dehydrogenases act on intermediates of branched-chain amino acid oxidation, isovalerylCoA and isobutyryl-CoA (15). All of these enzymes require ETF as an electron acceptor. The medium-chain enzyme is most active with fatty acids $8-10$ carbons in length, but also shows some activity with shorter and longer chain fatty acids and is, therefore, sometimes called general acyl-CoA dehydrogenase. Medium-chain $A C D$ activity is not required for oxidation of short-chain fatty acids, such as butyrate, or branched-chain ketoacids, such as alpha-ketoisocaproate. Absence of mediumchain ACD activity not only blocks the oxidation of mediumchain fatty acids, but also prevents the oxidation of long-chain fatty acids, such as palmitate, beyond the first few turns of the beta-oxidation cycle.

It should be noted that two of the other three reactions in the beta-oxidation cycle also involve enzymes that are specific for different chain lengths of fatty acid substrate: enoyl-CoA hydratase and beta-keto thiolase. A deficiency of one of the longerchain specific enzymes at either of these steps, rather than the ACD step, was considered unlikely for several reasons. The shortchain enoyl-CoA hydratase (crotonase) is relatively active with substrates up to 8-10 carbons long (12) and, therefore, would be expected to allow more normal rates of ketogenesis from octanoate than was found in the patients (Table 4). A deficiency of longer-chain thiolase activity is less readily excluded because the short-chain enzyme is highly specific for its four carbon substrate, acetoacetyl-CoA (21). A deficiency at either the thiolase or the enoyl-CoA hydratase step in beta-oxidation would be more likely to yield abnormal urinary organic acids with beta-keto or alpha, beta-unsaturated structure instead of the saturated dicarboxylic acids seen in our patients.

The present studies demonstrate that medium-chain ACD activity is deficient in liver tissue from affected patients. Studies of skin fibroblasts from these patients indicate that both the impairment in fatty acid oxidation and the defect in mediumchain ACD activity are expressed in these cells as well (6). This suggests that the enzyme deficiency may also be present in skeletal and cardiac muscle. The occurrence of the disorder in a brother and sister (patients B-1 and B-2), and the fact that parents are unaffected in both families are compatible with an autosomal recessive mode of inheritance. Preliminary studies in white blood cells support an autosomal recessive inheritance.

The metabolic defect in the three children reported here seems to be silent except under circumstances of prolonged fasting. This is compatible with the concept that, in the early stages of fasting adaptation, glucose continues to be the major oxidative fuel, whereas the utilization of fatty acids becomes important in later stages of fasting, beginning at about $12-16 \mathrm{~h}$ in infants and children. The development of episodic hypoglycemia in our three affected patients might be due to overutilization of glucose, underproduction of glucose, or a combination of the two during the stress of prolonged fasting. The modest elevations in blood lactate in one patient (M-1) suggest some abnormality in glucose metabolism, but could represent either defective hepatic gluconeogenesis or excessive glycolysis in peripheral tissues. A similar modest increase in fasting blood lactate has been noted in a patient with systemic carnitine deficiency (7). It should be noted, however, that the coma in these patients may not be entirely due to hypoglycemia; patient M-1 developed rather severe symptoms of vomiting and depressed consciousness while still normoglycemic. It is possible that these symptoms represent a toxic effect of the elevated levels of FFA or of fatty acid metabolites that accumulate as a result of the block of beta-oxidation. It is also 
noteworthy that, unlike patient $\mathrm{M}-1$, patient $\mathrm{B}-2$ tolerated fasting for $36 \mathrm{~h}$ without difficulty. The reason for this difference between M-1 and B-2 is unclear, but probably reflects differences in the degree of defect in fatty acid oxidation. It seems reasonable to expect that considerable heterogeneity will be seen in the clinical features of this disorder as other patients with medium-chain ACD deficiency are found.

An unexplained feature of the metabolic defect in our three patients is their abnormality in plasma and tissue levels of carnitine. In the non-fasted state, carnitine levels were low in both liver and skeletal muscle as well as in plasma. During fasting, with increased availability of FFA, there was an increase in plasma concentrations of carnitine, particularly of fatty acylcarnitine esters. It is clear that the impairment in hepatic fatty acid oxidation in these patients is not caused by carnitine deficiency. Instead, it seems likely that the abnormalities in carnitine are a consequence of the deficiency of medium-chain ACD activity, although the mechanism is not yet known.

There are similarities among our patients with medium-chain ACD deficiency, patients with dicarboxylic aciduria $(1,4,13$, 23,31 ), and cases of carnitine-unresponsive systemic carnitine deficiency $(11,16)$, which indicate that these are closely related or, perhaps, identical disorders. In all three, urinary $\mathrm{C}_{6}-\mathrm{C}_{10}$ dicarboxylic acids become markedly elevated during acute episodes of illness. In our patients with medium-chain ACD deficiency, these probably arise through cytosolic omega-oxidation of the medium-chain fatty acids that cannot be further oxidized in mitochondria. Similar reasoning has led other workers to postulate a deficiency of medium-chain ACD in some cases of dicarboxylic aciduria $(1,23)$. This raises the possibility that the dicarboxylic aciduria in patients thought to have systemic carnitine deficiency may not arise from omega-oxidation of longchain fatty acids, but might reflect a deficiency of medium-chain ACD activity. The major difference among these three disorders is the association of muscle weakness with systemic carnitine deficiency and the absence of apparent muscle weakness in our patients and those with dicarboxylic aciduria. But, as noted above, it is not unreasonable to suspect that muscle shares the defect in medium-chain ACD activity found in liver. If this is true, patients with muscle weakness may represent a more severe degree of enzyme deficiency whereas patients, such as ours and those with dicarboxylic aciduria, may have a less severe degree of deficiency that becomes manifest only under conditions of unusual stress.

Other investigators have pointed out the similarities betwen defects in fatty acid oxidation that involve the liver and Reye's syndrome $(4,18,27)$. Many of the features associated with Reye's syndrome were also seen in our patients with medium-chain ACD deficiency, including a biphasic prodrome of the illness (M-1), coma and hypoglycemia (M-1, B-1, B-2), vomiting (M$1)$, hepatomegaly (M-1), hyperammonemia (M-1), cerebral edema (B-1), and fatty liver (B-1). As defects in fatty acid oxidation become better defined, it is likely that an increasing number of patients considered to have Reye's syndrome will be recognized to have inborn errors of metabolism such as mediumchain ACD deficiency. The diagnosis of medium-chain ACD deficiency should now be much easier, because we (6) and, recently, Rhead and co-workers (25) have found that the defect is expressed in cultured skin fibroblasts. Our preliminary results indicate that white blood cells can also be used to detect affected individuals without the need for liver biopsy.

\section{REFERENCES AND NOTES}

1. Borg, L., Lindstedt, S., Steen, G., and Hjalmarson, O.: Aliphatic $\mathrm{C}_{6}-\mathrm{C}_{14}$ dicarboxylic acids in urine from an infant with fatal congenital lactic acidosis Clin Chim Acta 41:363 (1974)

2. Bougneres, P-F., Saudubray, J-M., Marsac, C., Bernard, O., Odievre, M., and Birard, J.: Fasting hypoglycemia resulting from hepatic carnitine palmitoyl transferase deficiency. J. Pediatr., 98: 742 (1981).

3. Carroll, J. E. Brooke, M. H. Devivo, D. C. Schumate, J. B., Kratz, R., Ringel S. P., and Hagberg, J. M.: Carnitine deficiency: lack of response to carnitine therapy. Neurology, 30:618(1980).
4. Chalmers, R. A., Lawson, A. M., Whitelaw, A., and Purkiss, P.: Twin siblings with a Reye's-like syndrome associated with an abnormal organic aciduria, hypoglycemia, diarrhea, and vomiting with close similarities to Jamaican vomiting sickness. Pediatr. Res., I4: 1097 (1980).

5. Chapoy, P. R., Angelini, C., Brown, W. J., Stiff, J. E., Shug, A. L., and Cederbaum S. D.: Systemic carnitine deficiency-a treatable inherited lipidstorage disease presenting as Reye's syndrome. N. Engl. J. Med., 303: 1389 (1980).

6. Coates, P., Hale, D. E., Stanely, C. A., Corkey, B. E., Hall, C. L., and Cortner J. A.: Genetic defect of medium-chain acyl $\mathrm{CoA}$ dehydrogenase activity impairs acid oxidation in human fibroblasts. (Submitted for publication)

7. DiDonato, S., Peluchetti, D., Rimoldi, M., Bertagnolio, B.. Uziel. G., and Cornelio, F.: Ketogenic response to fasting in human carnitine deficiencies. Clin. Chim. Acta, 100: 209 (1980)

8. DiMauro. S. and DiMauro, P. M.: Muscle carnitine palmityltransferase deficiency and myoglobinuria. Science, I82: 929 (1973).

9. Engel, A. G. and Angelini, C.: Carnitine deficiency of human skeletal muscle associated with lipid storage myopathy: a new syndrome. Science, $179: 899$ (1973).

10. Engel, A. G.: Possible causes and effects of carnitine deficiency in man. In: R. A. Frenkel and J. D. McGarry, Eds.: Carnitine Biosynthesis, Metabolism and Functions. pp. 271-284 (Academic press, New York, 1980).

11. Engel, A. G., Rebouche, C. J., Wilson, D., Glasgow, A. M., Romshe, C. A and Cruse R. P.: Primary systemic carnitine deficiency II: Renal handling of carnitine. Neurology (N.Y.), 31: 819 (1981).

12. Fong. J. C. and Schulz. H.: Short-chain and long-chain enoyl-CoA hydratases from pig heart muscle. In: J. M. Lowenstein. Ed.: Methods in Enzymology. Vol 71, Part C. pp. 390-398 (Academic Press, New York, 1981).

13. Gregerson, N., Rosleff, F., Kolaraa, S.. Hobolth, N.. Rasmussen. K., and Lauritzen, R.: Nonketotic $\mathrm{C}_{6}-\mathrm{C}_{10}$ dicarboxylic aciduria: biochemical investigation of two cases. Clin. Chim. Acta, 102: 179 (1980).

14. Hall, C. L.: Acyl-CoA dehydrogenases from pig liver mitochondria. In: J. M. Lowenstein, Ed.: Methods in Enzymology. Vol 71, Part C. pp. 375-383 (Academic Press, New York, 1981).

15. Ikeda, Y.. and Tanaka, K.: Isolation of 2-methyl-branched chain acyl-CoA dehydrogenase from rat liver mitochondria. Fed. Proc., 41: 1192 (1982).

16. Karpti, G.. Carpenter, S., Engel, A. G., Walters, G., Allen, J., Rothman, S. Klasson, G., and Mamer, O. A.: The syndrome of systemic carnitine deficiency. Neurology (Minneap.), 25: 16 (1975).

17. Krebs, H. A.: Rate of control of the tricarboxylic acid cycle. Adv. Enzy. Regul. 8: 335 (1970)

18. Mamer, O. A., Montgomery J. A., and Colle, E.: Profiles in altered metabolism III: (Omega-1)-hydroxyacid excretion in a case of episodic hypoglycemia. Biomed. Mass Spectrom., 7: 53 (1980).

19. McGarry, J. D. and Foster, D. W.: An improved and simplified radioisotopic assay for the determination of free and esterified carnitine. J. Lipid Res., I7. 277 (1976).

20. McGarry, J. D., Leatherman, G. F., and Foster, D. W.: Carnitine palmitoyltransferase I: The site of inhibition of hepatic fatty acid oxidation by malonylCoA. J. Biol. Chem., 253: 4128 (1978).

21. Middleton, B.: The oxoacyl-coenzyme A thiolases of animal tissues. Biochem. J., 132: 717 (1973)

22. Montagos, S., Genel, M., and Tanaka, K.: Ethylmalonic-adipic aciduria. J. Clin. Invest., 64: 1580 (1979).

23. Naylor, E. W., Mosovich, L. L., Guthrie, R., Evans, J. E., and Tiecklemann, $\mathrm{H}$ : Intermittent non-ketotic dicarboxylic aciduria in two siblings with hypoglycemia: an apparent defect in beta-oxidation of fatty acids. J. Inher. Metab. Dis., 3: 19 (1980).

24. Przyrembel, H., Wendel, U., Becker, K., Bremer, H. J., Bruinvis, L., Ketting, D., and Wadman. S. K.: Glutaric aciduria type II: Report on a previously undescribed metabolic disorder. Clin. Chim. Acta, 66: 227 (1976).

25. Rhead, W., Amendt, B., Fritchman, K., and Felts, S.: Dicarboxylic aciduria (DCA): deficiency of fatty acid beta-oxidation in intact fibroblasts and isolated fibroblast mitochondria and of the medium-chain acyl-CoA dehydrogenase (MCADH) in the mitochondrial matrix. Amer. J. Hum. Genet. 34: 61A (1982).

26. Rhead, W. J. and Tanaka, K.: Demonstration of a specific mitochondrial isovaleryl-CoA dehydrogenase deficiency in fibroblasts from patients with isovaleric acidemia. Proc. Natl. Acad. Sci., 77: 580 (1980).

27. Robinson, B. H., Oei, J., Sherwood, W. G., Slyper, A. H., Heininger, J., and Mamer, O. A.: Hydroxymethylglutaryl CoA lyase deficiency: features resembling Reye's Syndrome. Neurology, 30: 714 (1980).

28. Stanley, C. A. and Baker, L.: Hyperinsulinism in infancy: diagnosis by demonstration of abnormal response to fasting hypoglycemia. Pediatrics, 57: 702 (1976).

29. Stanley, C. A., Gonzales, E., and Baker, L.: Development of hepatic fatty acid oxidation in the newborn guinea pig. Pediatr. Res., 17: 224 (1983).

30. Sternlieb, I.: Electron microscopy of mitochondria and peroxisomes of human hepatocytes. In: H. Popper and F. Schaffuer, Eds.: Progress in Liver Disease. Vol. III. (Grune and Stratton, New York, 1979).

31. Truscott, R. J. W., Hick, I., Pullin, C., Halpern, B., Wilcken, B., Griffiths, H. Silink, M., Kilham, H., Grunseit, F.: Dicarboxylic aciduria: the response to fasting. Clin. Chim. Acta, 94: 31 (1979).

32. Williamson, J. R. and Corkey, B. E.: Assays of intermediates of the citric acid cycle and related compounds by fluorometric enzyme methods. In: J. M. Lowenstein Ed.: Methods in Enzymology. Vol 13. pp. 434 (Academic Press, New York, 1969).

33. The authors wish to thank the nurses of the Clinical Research Center and the 
house officers of the Children's Hospital of Philadelphia for providing excellent care of these patients: Joseph Egler for technical assistance: and Susan Anderson for helping with the manuscript. We also thank Dr. Camillus Witzleban for providing the information on liver histology: Dr. Stanton Segal for his advice: Dr. Albert Winegrad for many helpful discussions: and the Surgical Department of the Children's Hospital for their help in obtaining biopsies.

34. Requests for reprints should be addressed to: Charles A. Stanley, M. D., Div of Endocrinology/Diabetes, Children's Hospital of Philadelphia, 34th and
Civic Center Blvd.. Philadelphia, PA. 19104.

35. This work was supported in part by National Institutes of Health grants AM 15120, GM 25494. RR 00240, NS 17752, AM 07314, HD 07107, HD 08536 , GM 07511, a grant from the Juvenile Diabetes Foundation and the Medical Associates Research and Education Foundation of the Children's Hospital This work was presented in part at the May 1982 meeting of the Society for Pediatric Research.

36. Received for publication October 1, 1982

37. Accepted for publication March 17, 1983.

\title{
The Proportion of Mitochondrial Isoenzyme of Aspartate Aminotransferase Is Not Elevated in Reye's Syndrome
}

\author{
DONALD M. MOCK, ${ }^{(34)}$ MARION SCOTT, NELL I. MOCK, AND M. MICHAEL THALER \\ Division of Pediatric Gastroenterology and Nutrition, Department of Pediatrics, and the General Clinical \\ Research Center, University of California, San Francisco, California USA
}

\section{Summary}

We reexamined a previously reported, highly specific increase in the relative proportion of the mitochondrial isoenzyme of asparate aminotransferase (AST) in the serum of patients with Reye's Syndrome. Using ion exchange chromatography, we measured mitochondrial, cytosolic, and total AST in serum samples from (1) 10 patients early in the course of Reye's Syndrome; (2) nine controls with normal serum AST; and (3) seven controls with other diseases causing an increase in serum AST. The mitochondrial percentage $(2.8 \pm 2.0 \%)$ in Reye's Syndrome was significantly lower $(P<0.05)$ than that of both the normal control group $(6.1 \pm 7.1 \%)$ and the group with increased AST $(5.6 \pm 4.0 \%)$. We thus failed to confirm the previous report of a specific increase in the \% of mitochondrial isoenzyme in Reye's Syndrome, and conclude that the $\%$ of mitochondrial isoenzyme is not likely to be a useful marker of (or predictor for progression to) Reye's Syndrome.

\section{Abbreviations}

AST, aspartate aminotransferase

$\mathrm{LDH}$, lactate dehydrogenase

MDH, malate dehydrogenase

P-5-P, pyridoxal-5' -phosphate

Aspartate aminotransferase (EC 2.6.1.1, formerly SGOT) consists of distinct cytoplasmic and mitochondrial isoenzymes in most mammalian species including man $(2,6,11,18,19)$. In normal individuals, the $\%$ of total serum AST activity due to the mitochondrial isoenzyme has been reported to be less than $10 \%$ by investigators using the most precise techniques $(19,20)$. The $\%$ of mitochondrial serum AST generally remains relatively constant in liver diseases associated with hepatocellular damage, such as acute and chronic viral hepatitis, fulminant hepatitis, chronic active hepatitis, and cirrhosis $(7,9,13,27)$, but can be elevated acutely in response to hepatotoxins.
A rapid increase in the total serum concentration of AST occurs consistently in Reye's Syndrome (encephalopathy with fatty visceral degeneration), a grave complication of common childhood viral infections such as chicken pox and influenza $A$ and B (5). Swelling and pleomorphism of liver cell mitochondria occur early in the course of Reye's Syndrome and recede within days after onset $(1,15)$. The temporal pattern of these mitochondrial changes and the absence of other remarkable alterations in hepatocellular ultrastructure suggested the possibility that the early rise and rapid decline in serum AST in Reye's syndrome reflected release of isoenzyme from swollen mitochondria. Indeed, a previous report had detected such a rise in the mitochondrial isoenzyme (32). Because the mortality and morbidity of Reye's Syndrome can be reduced by early institution of control of intracranial pressure (28), a marker predicting which patients with influenza or varicella will progress to Reye's Syndrome would be of considerable clinical utility and could yield important information about onset, evolution, and pathogenesis of the syndrome. We sought to confirm and extend the previous observation in a group of well characterized patients with Reye's Syndrome. We applied highly accurate techniques for chromatographic separation and measurement of AST isoenzymes in 10 children with Reye's Syndrome, in seven children with other disorders causing an increase in serum AST, and in nine control subjects with normal serum AST. The $\%$ of mitochondrial isoenzyme was not elevated in any of the Reye's Syndrome patients.

\section{MATERIALS AND METHODS}

Assay of total AST activity. We used an enzyme-coupled spectrophotometric method for measurement of total AST activity in both serum samples and column eluates.

AST catalyzes the transfer of an amino group from aspartate to 2-oxoglutarate:

$$
\text { 2-oxoglutarate }+ \text { aspartate } \rightarrow \text { glutamate }+ \text { oxalacetate }
$$

In this assay the production of oxalacetate was coupled to oxi- 